\title{
Tibial Remodelling Douring Bone Defect Management Using Muthilevel Fragment Lengthening (Experimental Findings)
}

\author{
DOI: $10.17691 /$ stm2016,8.1.09
}

Received June 4, 2015.

\section{D.Y. Borzunov, MD, PhD, Deputy Director on Scientific Activities}

Russian Hizarov Scientific Center for Restorative Traumatology and Orthopaedics, Ministry of Healthcare of the Russian Federation, 6 M. Ulianova St., Kurgan, 640000, Russian Federation

The aim of the investigation was to reveal the features of tibial recovery during multilevel fragment lengthening for filling in an extensive bone defect in the conditions of maintained and disturbed intraosseous artery blood flow bed in the experiment.

Material and Methods. The experiment modeled the conditions of tibial bone defect filling by bi-level lengthening of the proximal and distal fragments in the conditions of a preserved and disturbed medullary blood flow. The experiment included 54 dogs divided into 4 groups. Radiographic, angiographic, histological, and statistical methods were used in the study.

Results. Changes in the architecture of the tibial vascularity net were not accompanied by rough hemodynamic circulatory disorders and depended on the period of the study and initial features of blood supply to the fragments. The conditions of the proximal fragment lengthening were more beneficial for medullary blood flow recovery and new bone formation. Both periosteal and endosteal bone structures took an active part in the distraction osteogenesis. Active periosteal osteogenesis resulted in formation of new bone layers on the periphery of the transported fragments in all the cases. Unified intraosseous nutrient artery vascular bed was formed six months after the external fixator removal. Distraction regenerates by multilevel lengthening of the distal tibial fragment were formed mainly due to the periosteal osteogenesis. Distal fragment lengthening featured a hypoplastic type of bone formation. No active bone tissue remodeling was observed in distal fragment lengthening. Periosteal layers of cancellous bone tissue were not identified on the entire periphery of bone fragments.

Conclusion. Prolonged disturbance in the major medullary blood flow occurs in multilevel lengthening of the distal fragment. Blood supply to the transported fragments is provided by periosteomedullary anastomoses. There is no complete recovery of the tibial nutrient artery net at 1.5-year follow-up.

Key words: bone defect; bi-level lengthening of bone fragments; intraosseous nutrient artery.

The llizarov non-free bone plasty is a gradual transport of an autologous graft within the bone defect which is surrounded and linked to the nearby tissues [1]. New bone tissue is generated by distraction osteogenesis in the defect that undergoes the remodeling process which is typical for bone tissue. Previous experimental studies at the Russian llizarov Center for Restorative Traumatology and Orthopaedics [2, 3] revealed that both the activity of tibial bone formation and the recovery of anatomical integrity and functions depended on the conditions of vascularity in the regeneration areas and on the intramedullary blood flow in the transported nonfree autologous grafts. Bone tissue possesses a great capacity for regeneration, and in theory any bone defect of any shape and length can be filled in with a new bone tissue applying the llizarov method bone fragment lengthening technology $[4,5]$. But in reality, extensive defects will need an extremely long time to be bridged if the llizarov bone plasty is applied. Moreover, the regenerated bone will most probably feature hypoplastic properties [6-10]. Therefore, the technologies were proposed for extensive defect management that provided several distraction regeneration sites during a single treatment stage that could remodel into bone tissue in a shorter period of distraction osteosynthesis [11].

The aim of the investigation was to reveal the features of tibial recovery during multilevel fragment lengthening for filling in an extensive bone defect in the conditions of maintained and disturbed intraosseous artery blood flow bed in the experiment.

Material and Methods. The experiment was conducted on 54 adult mongrel dogs. The experimental models were distal bone defects in groups $1(n=16)$ and $2(n=13)$ while proximal bone defects in the tibial metaphyses were formed in groups $3(n=12)$ and 4 $(n=13)$. The defects measured $20.9 \pm 0.3 \%$ from the initial tibial length. Proximal fragments were lengthened in the conditions of preserved intraosseous blood flow while the major flow was disturbed in the distal ones. Postresection defects were filled in using sequential bi-level formation of distraction regenerates in groups 1 and 3 (Figure 1 (a), (c)) while in groups 2 and 4 the lengthening ran simultaneously at two sites (Figure 1 (b), (d)).

In groups 1 and 2, corticotomies in the proximal fragment were produced at the level of the inferior edge of the tibial tuberosity and in its diaphyseal portion $(1.0-1.5 \mathrm{~cm}$ distally to the nutrient artery entrance into the bone marrow cavity). In groups 3 and 4, the corticotomies were performed in the metadiaphysis and diaphysis of the distal fragment. Therefore, the lengthening of the proximal fragment ran in the conditions of the preserved intraosseous blood blow while

For contacts: Dmitry Y. Borzunov, e-mail: borzunov@bk.ru 




Figure 1. Osteosynthesis diagrams: (a) group 1, sequential bi-level lengthening of the proximal fragment in the conditions of preserved intraosseous blood flow; (b) group 2, simultaneous bi-level lengthening of the proximal fragment in the conditions of preserved intraosseous blood flow; (c) group 3, sequential bi-level lengthening of the distal fragment in the conditions of disturbed intraosseous blood flow; (d) group 4, simultaneous bi-level lengthening of the distal fragment in the conditions of disturbed intraosseous blood flow

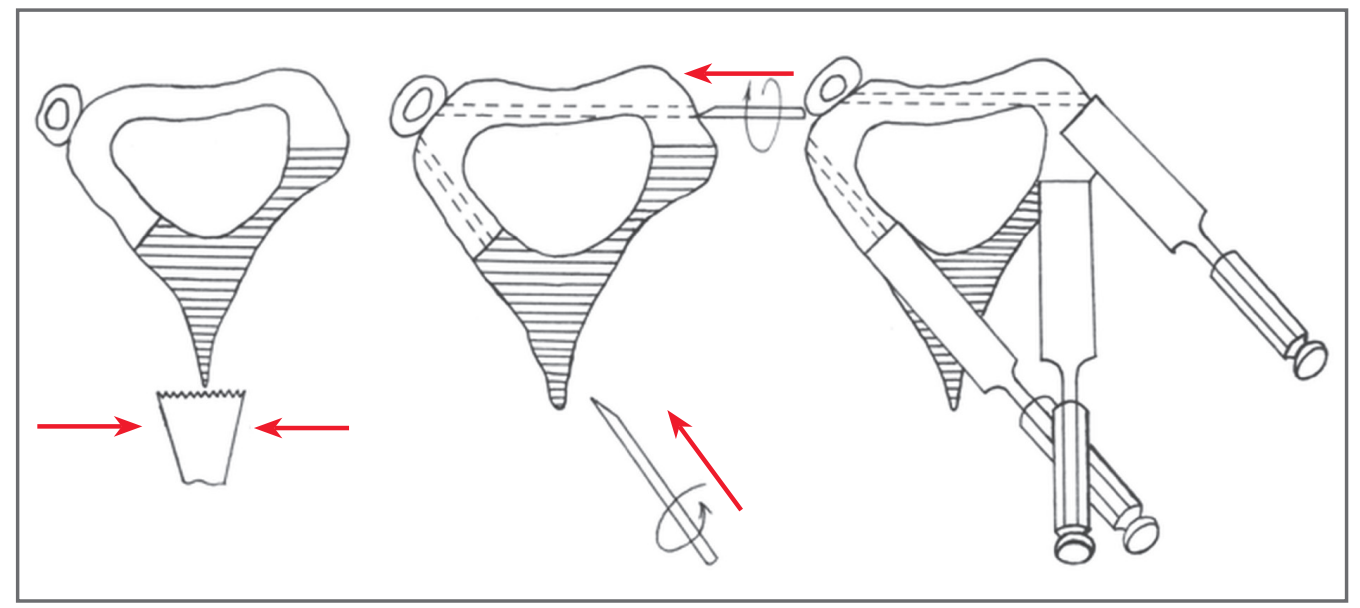

Figure 2. Diagram of partial corticotomy 
lengthening of the distal one was performed by disturbed major blood flow in the fragment under lengthening.

Additional corticotomies in groups 1 and 3 were produced after the non-free fragments had been transported to the half of the tibial defect cavity formed while in groups 2 and 4 the two corticotomies were produced at two levels simultaneously. Therefore, distraction regeneration was sequential in groups 1 and 3 while in groups 2 and 4 it was simultaneous. Bone transport of the fragments formed started six days after the operation with the rate $1 \mathrm{~mm}$ divided into 4 steps. Distraction at the level of the distal corticotomy in group 2 was initiated one day earlier.

Distraction continued until the lengthened tibial fragment docked with the opposite tibial fragment. Upon coaptation at the docking site, the conditions of rigid fragment fixation were produced. Longitudinal supportive compression of 0.5 to $1.0 \mathrm{~mm}$ followed every 7 or 10 days.

Fragment integrity was broken using a vibrant saw, chisel and by tangent wire insertion through the bone cortex along the line of a planned corticotomy without surgical instrument penetration into the bone marrow cavity (Figure 2).

Tibial vascular net was contrasted according to Gough [12].

Ethical principles of animal care (European Convention on Protection of Vertebral Animals used for Experimental and other Scientific Studies, adopted in Strasbourg on 18.03.1986, revised in Strasbourg on 15.06.2006) were strictly followed. The experiment was approved by the ethics committee of the institution and was performed in accordance with the principles of laboratory animal care as well as the national regulations on laboratory practice and the rules of studies with the use of experimental animals (Ministry of Health order No.755 dated 12.08.77).

\section{Results}

Distraction period. Distraction regenerates in groups 1 and 2 were formed both at the expense of both endosteal and periosteal osteogenesis. At the end of distraction in group 1, the proximal distraction regenerate partially lost its zonal structure and was formed mainly of cancellous bone tissue (Figure 3 (a), (b)). The distal regenerate had a connective tissue layer in the central part that was from 2 to $3 \mathrm{~mm}$ high (Figure 3 (c)). This connective tissue layer was composed from bundles of collagen fibers that lay densely, fibroblast cells, and capillaries. In several areas, it was crossed by bone trabeculae. At the end of fragments transport in group 2, both distraction gaps were filled in with distraction regenerates that maintained zonal structure. Periosteal bone layers on the transported fragments were observed in all the cases of groups 1 and 2 but were more expressed on the distal transported fragment (Figure $3(\mathrm{~d})$ ).

The radiographic vasograms of the tibia in groups 1 and 2 revealed an enhanced vascularity due to the widening of epipheaseal and metaphyseal arteries as well as intraosseuos nutrient artery net by the end of the period of bone fragments transport. The diameter of the vessels in the distraction regenerates of the proximal fragment in group 1 increased up to $414.3 \pm 52.0 \mu \mathrm{m}$ $(p<0.001)$ and of the distal one up to $323.5 \pm 14.2 \mu \mathrm{m}$ $(\mathrm{p}<0.05)$; in both fragments transported it increased respectively: in the proximal one up to $353.5 \pm 11.0 \mu \mathrm{m}$ $(p<0.001)$ and in distal one up to $385.0 \pm 20.1 \mu \mathrm{m}$ $(\mathrm{p}<0.001)$. It was associated with a prolonged staged fragments transport and presence of arterial hyperemia of the entire segment. Numerous arterial vessels that featured longitudinal direction were revealed in bone portions of the distal distraction regenerate (Figure $4(a)$ ).

Arteries of the bone portions had longitudinal
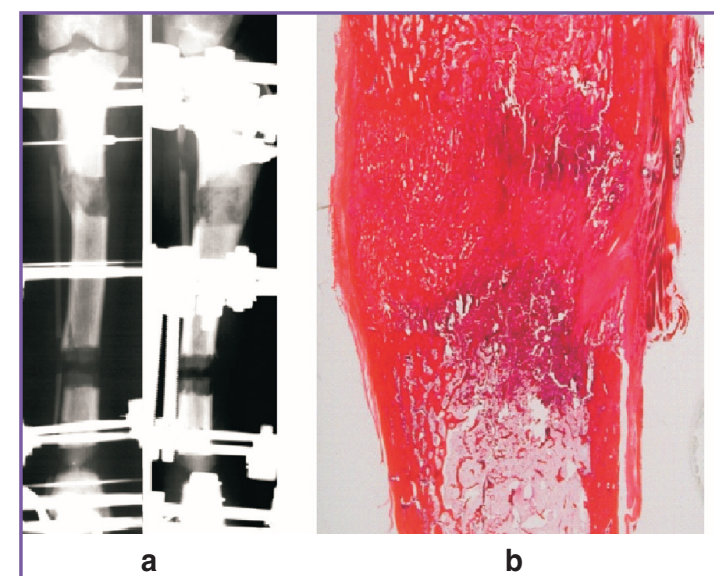

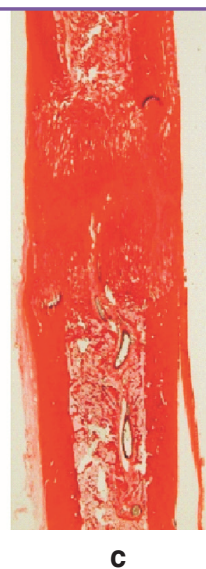

C

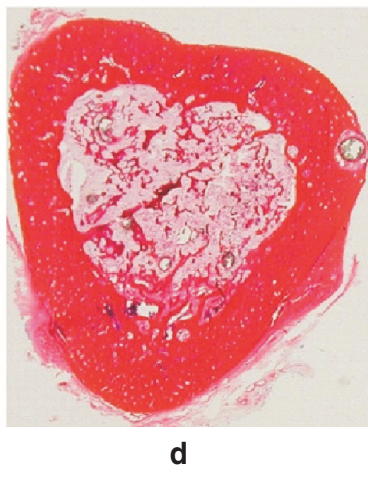

Figure 3. X-ray and histotopograms: (a) X-ray of the canine tibia in group 1 (21 days of distraction in the area of additional corticotomy, start of fixation of the distal distraction regenerate, 21 fixation days of the proximal distraction regenerate, 46 days of the experiment); (b)-(d) histotopograms: (b) proximal regenerate; (c) distal regenerate; (d) transverse section of the proximal transported fragment. Hematoxylin and eosin staining 


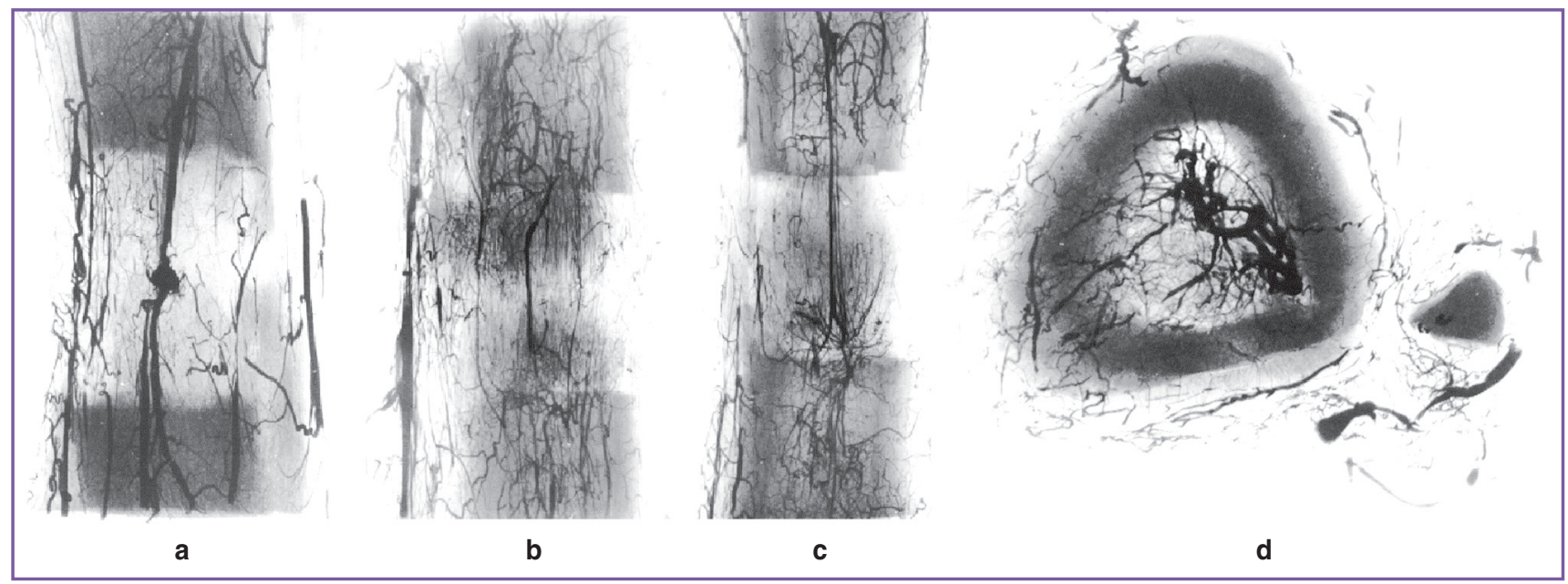

Figure 4. Fragments of radiographic vasograms of the distal distraction regenerate: (a) group 1 (25 distraction days in the area of an additional corticotomy, 53 days of the experiment); (b)-(d) group 2 (19 distraction days, 26 days of the experiment): (b) proximal regenerate, anteroposterior view; (c) distal regenerate, lateral view; (d) transverse section of the proximal transported fragment. Inverted image

orientation, reached the "growth zone" and crossed it in several areas (Figure 4 (b)). Major portion of the descending branch of the nutrient artery was visualized in the distal regenerate of group 2 dogs. The vascular net of the distraction regenerate was "poorer" though the new vessels that were oriented longitudinally were seen in both bone portions of the regenerate (Figure 4 (c)). The diameter of the vessels was $208.2 \pm 19.4 \mu \mathrm{m}$ $(p<0.001)$ that was considerably lower than the values in group 1 dogs at completion of fragments transport. The cortices of the fragments were penetrated with numerous periosteomedullary anastomoses (Figure 4 (d)).

In groups 3 and 4 , the radiographic vasograms taken before the initiation of distraction showed a maintained blood supply to the distal lengthened fragment through a shunting periosteal net of the tibia. Medullary-toperiosteal anastomoses functioned on the fragment periphery that penetrated into the cortical layers of the fragments formed. The periosteal vascular net had mainly a longitudinal direction of the flow. The anastomoses were curved and were mainly oriented in the transverse direction.

The distraction regenerates in groups 3 and 4 were formed mainly at the expense of the periosteal osteogenesis. In the majority of the cases, the area of the interfragmentary gaps was greater than the area of the distraction regenerate formed. In other words, a hypoplastic type of bone formation prevailed in lengthening of the distal fragment. At the end of the fragments transport, both diastases were filled in with distraction regenerates that featured zonal structure. In all the cases, the light zone in the proximal regenerate measuring from 2 to $8 \mathrm{~mm}$ was positioned in the center. In the majority of the cases, the light zone in the distal regenerate was shifted to the distal transported fragment (Figure 5 (a), (b)). The transported fragments had porous cortical layers that featured numerous resorption cavities that were filled in with necrotic content. Mainly osteoclasts were present in the cavities and vascular canals; however active osteoblasts were also encountered. Periosteal cancellous bone layers did not cover the entire peripheries of the fragments. Their thickness did not exceed $1.0 \mathrm{~mm}$ (Figure 5 (c)).

The findings of radiographic vasograms in groups

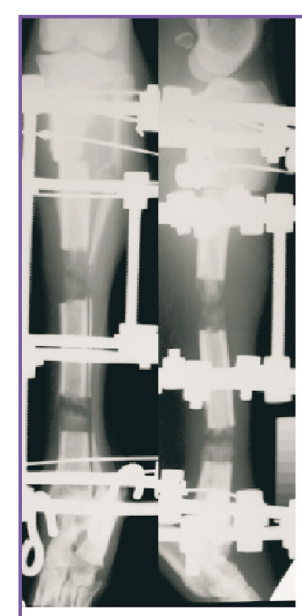

a



b



c

Figure 5. X-ray and histotopograms: (a) X-ray of tibia, group 4 (end of distraction, 26 days of the experiment); (b)-(c) histotopograms, group 3: (b) distal regenerate (end of distraction, 47 days of the experiment); (c) transverse section of the distal transported fragment (end of distraction, 47 days of the experiment). Hematoxylin and eosin staining 
3 and 4 by the end of distraction revealed that blood supply to the transported fragments was maintained through the functioning of the periosteomedullary anastomoses. The periosteal vascular net of the tibia was widened. The picture of vascularity was more expressed in the proximal tibial epimetaphysis and proximal transported fragment. Newly formed vessels were not numerous in the distraction regenerates. The arteries of the new bone portions were mainly oriented longitudinally, reached the central part of the distraction regenerates and crossed it in several areas. Upon completion of distraction, the proximal distraction regenerate had newly formed vessels which diameter measured 340.0 $\pm 14.0 \mu \mathrm{m}$ ( $p>0.05)$. In the same period, the vessels of the distal regenerate had a diameter of $315.0 \pm 13.0 \mu \mathrm{m}(p>0.05)$. The widening of the arteries of the periosteal net of the tibia was accompanied by their winding and retrograde orientation relative to the blood flow. The vessels in the transported fragments of both groups were randomly oriented at this point of the experiment.

Fixation period. Blood supply to bone fragments of groups 1 and 2 in this period ran through the bed of the intraosseous nutrient artery and periosteal net (Figure 6 (a)). Widened medullary-to-periosteal anastomoses continued their functioning in the cortical layers. The architecture of the vascular bed in the proximal bone part was presented by a delicate net of numerous newly formed vessels that were oriented longitudinally and crossed the connective tissue layer. The vascular picture of the distal part of the regenerate consisted of the vessels that divided in dichotomous and trichotomous ways that were not numerous (Figure 6 (b)).

Mean diameter of the newly formed vessels in the proximal regenerate of group 1 decreased down to $255.3 \pm 27.5 \mu \mathrm{m}(p<0.01)$ and in the distal one down to $278.5 \pm 29.2 \mu \mathrm{m}(\mathrm{p}>0.05)$. It was associated with a gradual recovery of hemocirculation in the bone marrow cavity

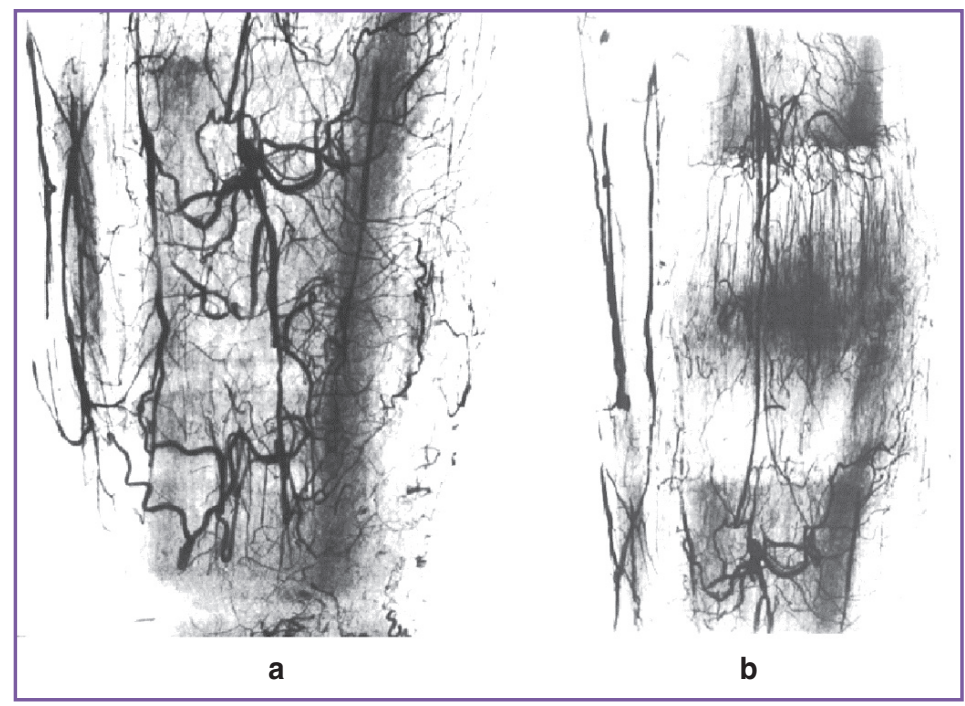

Figure 6. Fragments of radiographic vasograms of the tibia in group 1 (30 fixation days of the distal distraction regenerate, 85 days of the experiment): (a) anteroposterior view of the distal transported fragment; (b) anteroposterior view of the distal regenerate. Inverted image

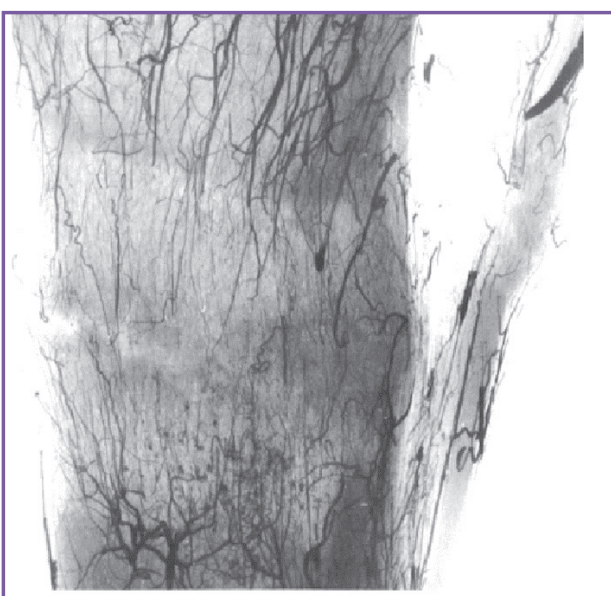

a

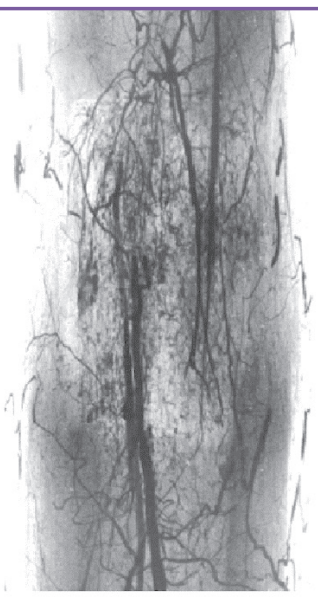

b

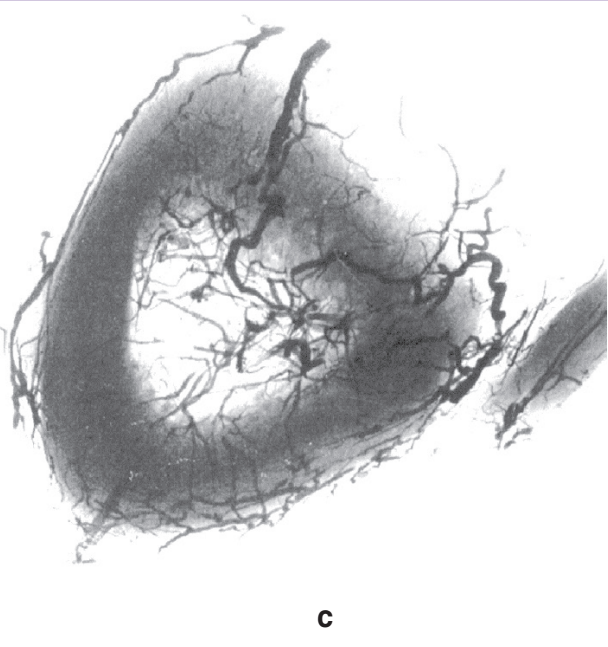

Figure 7. Fragments of tibial radiographic vasograms in group 2 (58 fixation days, 62 days of the experiment): (a) proximal regenerate; (b) distal regenerate, lateral views; (c) cross section of the proximal transported fragment. Inverted image 
of the tibia due to merged vessels in the bone parts of regenerates and due to the reduction in the vessel diameter in the fragments as compared with the period of fragments transport completion.

Hemocirculation disorders in the transported fragments deteriorated to some extent by simultaneous bi-level lengthening of the proximal tibial fragment in group 2 as compared with the respective values in group 1. Moreover, a significant widening of vessels up to $278.5 \pm 23.2 \mu \mathrm{m} \quad(p<0.05)$ and $284.5 \pm 22.7 \mu \mathrm{m} \quad(\mathrm{p}<0.01)$, respectively in the transported fragments was noted. Histological study in the fixation period found that the proximal regenerate consisted of predominantly cancellous bone tissue that had different stages of maturity. The newly formed bone tissue that was located closer to the fragments had features of lamellar bone that was undergoing partial resorption. Foci of connective tissue were seen in the intermediary space. The distal regenerate consisted of



a

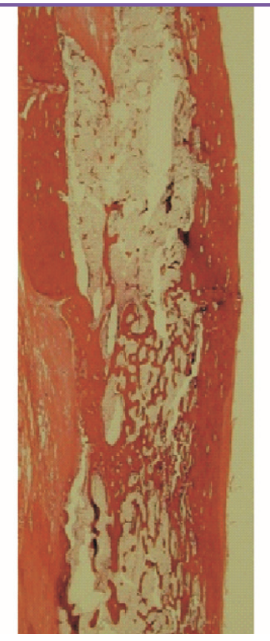

b

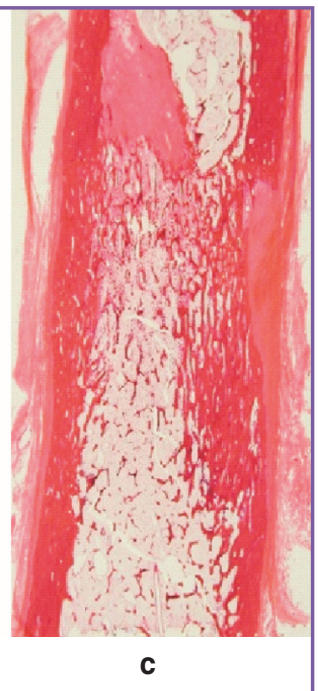

Figure 8. X-ray and histotopograms: (a) X-ray of the tibia in group 4 (75 fixation days, 105 experimental days); (b)-(c) histotopograms: (b) proximal regenerate; (c) distal regenerate. Hematoxylin and eosin staining cancellous bone tissue. Hematopoietic fatty bone marrow was present in the intertrabecular spaces.

By the end of fixation period, the osteogenesis process in groups 1 and 2 was accompanied by the recovery of the major blood flow in the system of the intraosseous nutrient artery. The vasograms of the tibia showed "impoverishment" of the vascularity picture due to reduction in the number of medullary-toperiosteal anastomoses and absent contrasting of the vessels of the $4^{\text {th }}$ and $5^{\text {th }}$ row. The visual picture was confirmed by the findings obtained. The vessels of the proximal fragment had a mean diameter of $246.4 \pm 13.5 \mu \mathrm{m}$ $(p>0.05)$ and the ones in the proximal fragment measured 256.6 $\pm 19.4 \mu \mathrm{m}(p>0.05)$. The vascular net of the newly formed bone parts was presented by numerous arterial vessels of different sizes with the preserved longitudinal orientation (Figure 7). Mean diameter of the proximal regenerate decreased to $229.1 \pm 17.5 \mu \mathrm{m}(p<0.05)$ and the one of the distal regenerate was $231.8 \pm 15.1 \mu \mathrm{m}(p<0.01)$.

By the end of fixation period in groups 3 and 4 , the medullary blood flow in the non-free fragments and newly formed bone parts started its formation along the intraosseous bed of the nutrient artery. The transported fragments retained blood supply from the periosteal net of the tibia that was provided by the functioning of the periosteomedullary anastomoses. The histological study revealed arteries that were empty or had plasma mixed with erythrocytes in their lumen. Periosteal vessels still prevailed in the newly formed vascular net of distraction regenerates that shunted those fragments with participation of the re-canalization of the vascular bed of the nutrient artery of the fragments' bone marrow cavity. Moreover, there was a change in the architecture of the vascular bed of the intraosseous artery. Several branches of $2^{\text {nd }}$ and $3^{\text {rd }}$ rows lost the dominating longitudinal direction. Their winding net was shunted by periosteal vessels. Solitary vessels with the mean diameter of $319.0 \pm 21.0 \mu \mathrm{m}(p>0.05)$ in the proximal distraction regenerate and of $321.0 \pm 21.0 \mu \mathrm{m}(p>0.05)$ in the distal one were contrasted.

The formation of distraction regenerates was of hypoplastic type. The area of the interfragmentary gaps was larger than the area of the newly formed bone tissue (Figure 8 (a)). The distraction regenerates had cortical layers but those featured bone defects that were filled with fibrous tissue (Figure 8 (b), (c)).

Period after the apparatus removal. This period was characterized by further remodeling of the arterial net of the tibia. The contrast mass filled in the descending and ascending branches of the nutrient artery. The terminal arteries of the descending branch could be followed on the entire extension of the fragments, distraction regenerates and fragments docking areas. Partial reduction of periosteomedallary anastomoses started in groups 1 and 2 .

The state of a widened arterial periosteal bed in the tibia was more prolonged in groups 3 and 4 after the removal of the apparatus. Blood supply to the bony parts of the restored segment was supported predominantly by the net of periosteomedullary anastomoses which was not numerous. It should be noted that a long-term absence of the reduction in anastomoses that shunted the fragments was observed.

Discussion. As stated Trueta [13], a lot of important issues of the osteogenesis process remain unclear. However, he was sure that the osteogenetic blood vessels are the organizers of bone formation. They form the matrix for syncytial cells and their combinations that determine bone architecture.

The experiment conducted revealed that multilevel lengthening of the proximal fragment used for filling a tibial bone defect ran in beneficial conditions for 
recovery of the anatomic and functional integrity of the intraoseeous arterial net. The results that were obtained are in accordance with the studies that were previously reported and were dedicated to investigation of osteo- and angiogenesis by llizarov management of a long bone defect of a similar large extension [2]. However, there are controversial studies that reported decompensation disorders in blood circulation in the distal parts of the limb when bone defects constituted $15 \%$ of the initial segment length and were managed with the classical llizarov technologies $[14,15]$.

The process of osteogenesis by bi-level lengthening of the proximal tibial fragment ran in the conditions of arterial hyperemia in the operated segment. In their previous works, our colleagues observed an increased blood flow to the limb tissues during distraction osteogenesis [16]. According to our findings, multilevel low traumatic break of the integrity of a tibial fragment to be lengthened did not block the blood flow along the bed of the intraosseous nutrient artery to the fragments formed. The arterial net of fragments got filled up at all stages of the experiment and the medullary blood flow was maintained. Widening of the arterial bed had a compensation character. It was probably associated with the difficulties of the venous outflow from the fragments that were transported and with temporary disorders of the intraosseous blood circulation. Recirculation in the transported fragments was provided by remodeling of the periosteal vascular net that featured formation of meduallary-to-periosteal anastomoses that supported the major blood supply to the fragments and redistributed blood flow. The change in the vascular net architecture of the tibia was not accompanied by severe circulatory disorders in hemodynamics and depended on the period of observation. The quantitative assessment of the vascular flow of the tibia allowed us to obtain objective findings in the changes of the intraosseous vascular net. The conditions in groups 1 and 2 were more beneficial for the recovery of the medullary blood flow. Remodeling of the intraosseous vascular bed of the tibia was manifested by the widening of the terminal parts of the nutrient artery branches and periosteal vessels, by activity of periosteomedullary anastomoses in the conditions of functional loading on the vascular system of the segment under lengthening. It served the adequate blood supply to non-free fragments and newly formed bone parts. Participation of the bone marrow cavity vascular net prevailed in the process of new vessels formation in the distraction regenerates. The partial reduction of the periosteomedullary anastomoses appeared not earlier than six months after the apparatus had been removed and a unified vascular bed of the recovered intraosseous nutrient artery had been formed. An initial prolonged disturbance of the major medullary blood flow was characteristic in the management of post-resection bone defects with a multilevel lengthening of the distal fragment. Blood supply to the tibial fragment under lengthening was supported by the activity of the periosteomedullary anastomoses. The filling of the arterial lumens in the fragments with plasma probably hindered vascular bed obliteration and later provided the recovery of the major blood flow during the remodeling of the tibial nutrient artery net. No reduction of the arterial shunts was observed at the long-term follow-up (1.5 years). The remodeling of the vascular bed of the nutrient artery was characterized by the changes of its architecture. The vascular net was oriented not only in the longitudinal direction but also a retrograde orientation of the arterial vessels was formed in several areas.

Conclusion. A prolonged disturbance in the major medullary blood flow occurs during the process of multilevel lengthening of the distal tibial fragment. Blood supply to the transported fragments is supported by the activity of the periosteomedullary anastomoses. Complete remodeling of the tibial nutrient artery net was not revealed at 1.5 year follow-up.

Study Finding. The experiment was conducted in the frames of the budget state support for scientific research.

Conflicts of Interest. The author declares that there no conflicts of interest.

\section{References}

1. llizarov G.A. Sposob zameshcheniya defekta dlinnoy trubchatoy kosti [Method of replacing a long bone defect]. Inventor's Certificate No.1124269/31-1. 1971.

2. Larionov A.A. Vascularization of tibia when compensating diaphyseal defect by elongation of one of the fragments according to G.A. Ilizarov's method. Arkhiv anatomii, gistologii i embriologii 1989; 11: 21-27.

3. Shevtsov V.I., Borzounov D.Y., Petrovskaya N.V., Osipova E.V. The characteristic features of the reorganization of tibial arterial bed for filling the defect of leg bones using the technique of multilevel lengthening of the proximal fragment (experimental study). Geniy ortopedii 2005; 2: 5-13.

4. Ilizarov G.A. Transosseous osteosynthesis. Theoretical and clinical aspects of the regeneration and growth of tissue. Springer-Verlag-Heidelberg 1992; 802 p., http://dx.doi. org/10.1007/978-3-642-84388-4.

5. Green S.A., Jackson J.M., Wall D.M., Marinow H., Ishkanian J. Management of segmental defects by the llizarov intercalary bone transport method. Clin Orthop 1992; 280: 136142, http://dx.doi.org/10.1097/00003086-199207000-00016.

6. Paley D., Maar D.C. Ilizarov bone transport treatment for tibial defects. J Orthop Trauma 2000; 14(2): 76-85, http://dx.doi. org/10.1097/00005131-200002000-00002.

7. El-Alfy B., El-Mowafi H., El-Moghazy N. Distraction osteogenesis in management of composite bone and soft tissue defects. Int Orthop 2010; 34(1): 115-118, http://dx.doi. org/10.1007/s00264-008-0574-3.

8. Smith W.R., Elbatrawy Y.A., Andreassen G.S., Philips G.C., Guerreschi F., Lovisetti L., Catagni M.A. Treatment of traumatic forearm bone loss with llizarov ring fixation and bone transport. Int Orthop 2007; 31(2): 165-170, http://dx.doi. org/10.1007/s00264-006-0172-1.

9. Liodakis E., Kenawey M., Krettek C., Wiebking U., Hankemeier S. Comparison of 39 post-traumatic tibia bone transports performed with and without the use of an intramedullary rob: the long-term outcomes. Int Orthop 2011; 35(9): 1397-1402, http://dx.doi.org/10.1007/s00264-010-1094-5. 
10. Kocaoglu M., Eralp L., Rashid H.U., Sen C., Bilsel K. Reconstruction of segmental bone defects due to chronic osteomyelitis with use of an external fixator and an intramedullary nail. J Bone Joint Surg Am 2006; 88(10): 2137-2145, http:// dx.doi.org/10.2106/JBJS.E.01152.

11. Borzunov D.Y. Long bone reconstruction using multilevel lengthening of bone defect fragments. Int Orthop 2012; 36(8): 1695-1700, http://dx.doi.org/10.1007/s00264-012-1562-1.

12. Gough J.A. A method of injecting the blood-vessels for roentgenological studies and simultaneously embalming. The Anatomical Record 1920; 18(2): 193-203, http://dx.doi. org/10.1002/ar.1090180209.

13. Trueta J., Buhr A.J. The vascular contribution to osteogenesis. V. The vasculature supplying the epiphysial cartilage in rachitic rats. J Bone Joint Surg $\mathrm{Br}$ 1963; 45: 572-581.

14. Yang L., Li Q., Zhou Z. Changes of blood circulation of the extremity with tibia shaft defects treated with external fixation. Zhonghua Wai Ke Za Zhi 2000; 38(2): 145-147.

15. Yang L., Li Q., Zhou Z. Changes of blood circulation of the extremity after external fixation for tibia shaft defect: an experimental study. Chin J Traumatol 2001; 4(2): 89-92.

16. Shevtsov V.I., Gordievskikh N.I., Bunov V.S., Petrovskaya N.V. Changes in blood flow during tibial thickening by the llizarov method. Bull Exp Biol Med 2002; 134(6): 525527, http://dx.doi.org/10.1023/a:1022988423449. 